\title{
Preparation and Integration of ALHAT Precision Landing Technology for Morpheus Flight Testing
}

\author{
John M. Carson III ${ }^{1,2, *}$ Edward A. Robertson ${ }^{1, \dagger}$, Diego F. Pierrottet ${ }^{4, \ddagger}$ \\ Vincent E. Roback ${ }^{3, \S}$, Nikolas Trawny ${ }^{2, \boldsymbol{\uparrow}}$, Jennifer L. Devolites ${ }^{1, \|}$ \\ Jeremy J. Hart ${ }^{1, * *}$ Jay N. Estes ${ }^{1, \dagger \dagger}$, Gregory S. Gaddis ${ }^{5, \ddagger \ddagger}$ \\ ${ }^{1}$ NASA Johnson Space Center, $\quad{ }^{2}$ Jet Propulsion Laboratory, California Institute of Technology \\ ${ }^{3}$ NASA Langley Research Center, $\quad{ }^{4}$ Coherent Applications, Inc. ${ }^{5}$ NASA Kennedy Space Center
}

\begin{abstract}
The Autonomous precision Landing and Hazard Avoidance Technology (ALHAT) project has developed a suite of prototype sensors for enabling autonomous and safe precision landing of robotic or crewed vehicles on solid solar bodies under varying terrain lighting conditions. The sensors include a Lidar-based Hazard Detection System (HDS), a multipurpose Navigation Doppler Lidar (NDL), and a long-range Laser Altimeter (LAlt). Preparation for terrestrial flight testing of ALHAT onboard the Morpheus free-flying, rocket-propelled flight test vehicle has been in progress since 2012, with flight tests over a lunar-like terrain field occurring in Spring 2014. Significant work efforts within both the ALHAT and Morpheus projects has been required in the preparation of the sensors, vehicle, and test facilities for interfacing, integrating and verifying overall system performance to ensure readiness for flight testing. The ALHAT sensors have undergone numerous stand-alone sensor tests, simulations, and calibrations, along with integrated-system tests in specialized gantries, trucks, helicopters and fixed-wing aircraft. A lunar-like terrain environment was constructed for ALHAT system testing during Morpheus flights, and vibration and thermal testing of the ALHAT sensors was performed based on Morpheus flights prior to ALHAT integration. High-fidelity simulations were implemented to gain insight into integrated ALHAT sensors and Morpheus GN\&C system performance, and command and telemetry interfacing and functional testing was conducted once the ALHAT sensors and electronics were integrated onto Morpheus. This paper captures some of the details and lessons learned in the planning, preparation and integration of the individual ALHAT sensors, the vehicle, and the test environment that led up to the joint flight tests.
\end{abstract}

\footnotetext{
*ALHAT SE\&I Lead, IPA Detail to JSC from JPL, AIAA Associate Fellow.

${ }^{\dagger}$ ALHAT Deputy Project Manager.

¥ALHAT NDL and LAlt Systems Engineer, AIAA Member.

$\S$ ALHAT Flash Lidar Systems Engineer, AIAA Senior Member.

『ALHAT HDS Lead, AIAA Member.

$\|$ Morpheus SE\&I Lead.

** Morpheus GN\&C Lead.

${ }^{\dagger}$ Morpheus Vehicle Manager.

$\ddagger \ddagger$ Morpheus/ALHAT KSC Site Manager.

(C)2014. All Rights Reserved.
} 


\section{Introduction}

The Entry, Descent and Landing (EDL) phase of a spacecraft lander mission is a high risk event that is traditionally tested at the integrated-system level only once - during the actual landing event. The traditional development and flight-implementation path for new EDL GN\&C technologies has been to develop and test at the component level within specialized testbeds and high-fidelity simulations. The testbeds are often specialized gantries, helicopters, and/or aircraft that provide some degree of flight-like dynamic motion in which real-time GN\&C hardware and software performance can be assessed in open-loop operation. The development of free-flying, suborbital rocket vehicles, as referred to as Vertical Testbeds (VTBs), has provided a new capability for developing, maturing and infusing new EDL GN\&C technologies into missions at an accelerated pace. These VTBs provide the capability of testing new GN\&C technologies within more flightrelevant dynamics to assess real-time performance. Additionally, VTBs provide a unique capability to test and refine these technologies as a fully integrated system in closed-loop operation well in advance of launch.

The NASA Autonomous precision Landing and Hazard Avoidance Technology (ALHAT) project was chartered in 2006 with developing new GN\&C technologies in sensors and algorithms for crewed or robotic landers that enable autonomous and safe precision landing onto the lunar surface under any terrain lighting conditions. ${ }^{1-3}$ The project and teaming has been a highly-successful demonstration of multi-center collaboration within NASA, involving team members from NASA Johnson Space Center (JSC), NASA Jet Propulsion Laboratory (JPL), NASA Langley Research Center (LaRC), and many other supporting contractors and research centers, including the Charles Stark Draper Laboratory (CSDL) and the Johns Hopkins Applied Physics Laboratory (APL). The ALHAT team has developed several generations of prototypes sensors and algorithms that provide precision velocimetry and altimetry, Hazard Detection (HD) ${ }^{4}$ and Hazard Relative Navigation (HRN), and Terrain Relative Navigation (TRN).$^{5-8}$ The current-generation sensor suite includes of a flash Lidar (Light Detection and Ranging) ${ }^{9}$ based Hazard Detection System (HDS), ${ }^{10}$ a Navigation Doppler Lidar (NDL), ${ }^{11}$ and a long-range Laser Altimeter (LAlt). ${ }^{12}$ These sensors and algorithms have been tested in dedicated labs, on specialized gantries, and within helicopters and fixed-wing aircraft to evaluate performance, determine necessary revisions, and step toward next-generation implementations. ${ }^{13-15}$ What remains for the current generation of ALHAT technology is to demonstrate and verify autonomous and safe precision-landing capabilities in closed loop on a VTB platform. This verification will raise the Technology Readiness Level (TRL) of ALHAT to a 6 , which signifies the techniques and approaches for the ALHAT system have been operationally demonstrated in a relevant flight-like environment - a necessary step toward infusion into future spaceflight applications.

The NASA Morpheus project began in 2010 as an effort to design, develop, implement and test new lander technologies at low cost and at an accelerated pace. From engine ignition through landing, the Morpheus vehicle is a fully autonomous VTB with no remote piloting. The current-generation vehicle includes provisions for testing ALHAT and ALHAT-like GN\&C technologies, and incorporates new propulsion technology based on liquid oxygen (LOX)/methane, as well as smaller technology test capabilities in structures and avionics. The vehicle Flight Software (FSW) is based on the NASA Core Flight Software (CFS). The vehicle GN\&C architecture implements a dual-string Navigation (Nav) capability to provide a nominal terrestrial VTB GPS-based Nav, as well as an experimental Nav (i.e., ALHAT Nav herein) for progressively testing and maturing new technologies from initial open-loop testing through fully closed-loop tests. The Morpheus team is also a multi-center collaboration within NASA, involving NASA-JSC, NASA Marshall Space Flight Center (MSFC), NASA Goddard Space Flight Center (GSFC), NASA Stennis Space Center (SSC), NASA-KSC and NASA Glenn Research Center (GRC), along with many supporting contractors.

The flight testing of the ALHAT sensor suite onboard Morpheus was conducted in Spring 2014, and detailed performance analyses are underway. The preparation of the ALHAT sensor and the integration activities leading up to the joint Morpheus/ALHAT flight testing was a tremendous multiyear joint effort between the ALHAT and Morpheus teams. This paper focuses on these stand-alone and integrated-systems efforts that led to the flight tests. Sections II and III give a high-level overview of the ALHAT concept of operations and the current-generation ALHAT sensors, respectively. Some historical details on the development and testing with the stand-alone sensors is provided in Section IV as this work was critical in the refinement of the sensor measurements and algorithms in preparation for flight tests. Section V provides 
insight into the simulation environments developed to understand integrated flight testing and to refine sensor and GN\&C algorithm parameters to achieve desired performance. Environmental considerations (both vehicle induced and local test conditions) are discussed within Section VI: these include such conditions as the vehicle thermal and vibration environment, atmospheric particulates and humidity, and development of the ground terrain for the testing. Details on the final integration and functional testing of the ALHAT sensor on Morpheus is discussed in Section VII which led up to the first joint tether and flight testing, which was conducted on a lunar-like terrain field constructed at NASA KSC. Finally, several closing remarks are made in Section VIII to capture some of the many lessons learned throughout the development of ALHAT and the collaboration with Morpheus.

\section{Overview of ALHAT Operations}

The ALHAT system was designed to accomplish three primary requirements: provide autonomous and safe, precision landing capabilities under any terrain lighting conditions; provide global precision landing to within 90-meters $(3 \sigma)$ of an a priori defined Intended Landing Point (ILP); and, provide local precision landing to within 3 meters $(3 \sigma)$ of a safe landing site determined in real time within a 90-meter $\times 90$-meter region surrounding the ILP. These requirements are accomplished by implementing ALHAT sensors and ALHAT Nav within a lander's closed-loop GN\&C system to perform several phases of descent, as illustrated in Figure 1: Terrain Relative Navigation (TRN), Hazard Detection and Avoidance (HDA), and Hazard Relative Navigation (HRN).

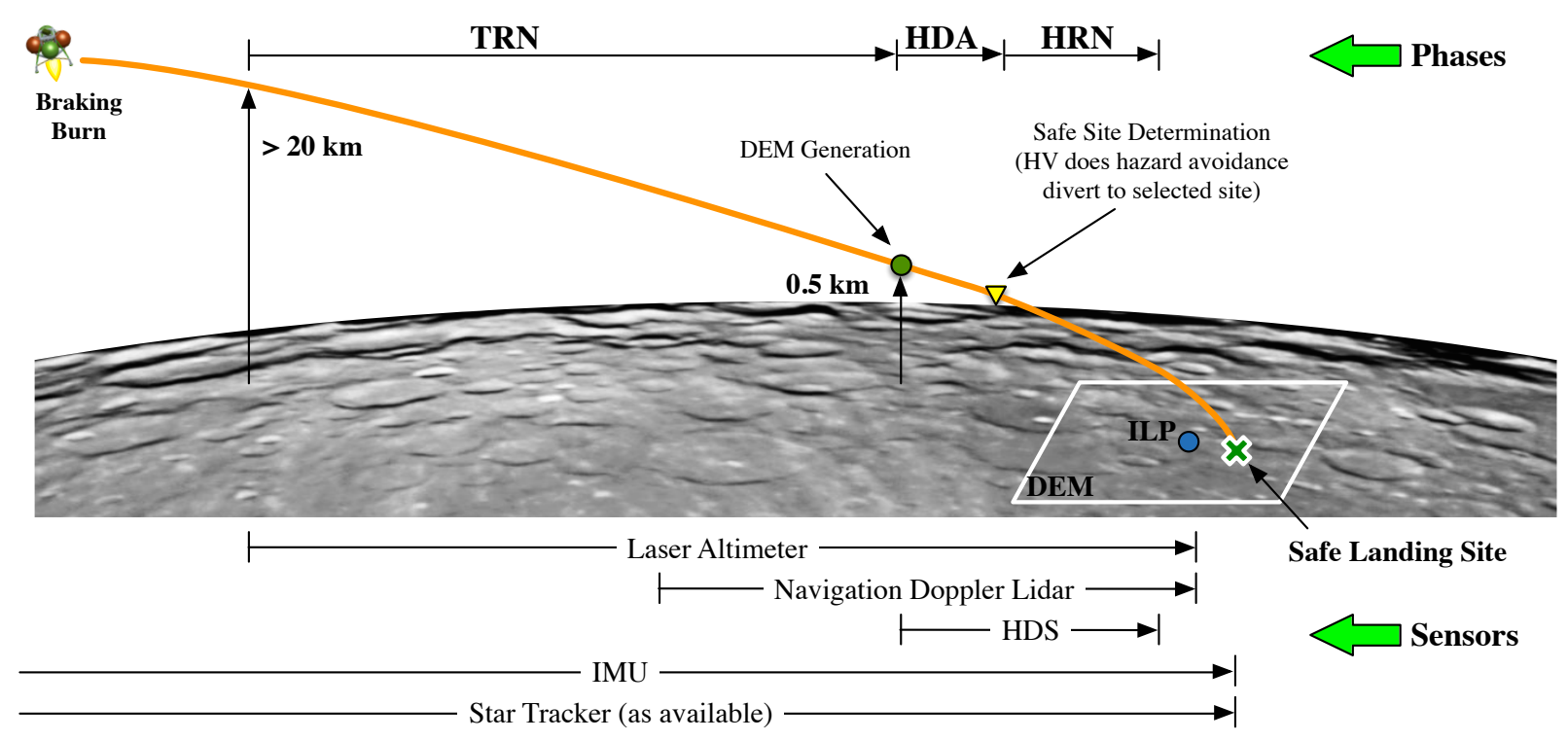

Figure 1. Phases of ALHAT operation for enabling safe precision landing. Approximate altitudes for phases are shown, along with typical timelines for various sensors being processed within ALHAT Nav.

The ILP is a guidance target location on the surface of the planet or moon that is determined prior to EDL (and likely pre-mission) based on orbital reconnaissance imagery and overall mission objectives. To provide the required global landing precision, the ALHAT system first implements TRN to localize the descending spacecraft relative to the ILP, and onboard GN\&C algorithms make use of this information to determine thrust or RCS (Reaction Control System) corrections to reduce position error. The ALHAT project investigated several techniques for TRN: active laser or Lidar based ${ }^{5,6}$ and passive optical based. ${ }^{7,8}$ Both active and passive TRN approaches were found to provide comparable precision. For landings in the dark or in unlit terrain such as the lunar south pole, active TRN sensors are required.

Since TRN makes use of reconnaissance imagery that has a finite and generally coarse $(5+$ meter per 
pixel) resolution, a higher-resolution map must be generated to determine both a safe landing site and for providing the local required landing precision. Most robotic and crewed vehicles have hazard tolerances those pose landing risks that cannot be fully resolved by using coarse orbital imagery, which drives most missions to target benign and statistically safe landing areas on the Moon and Mars. During the HDA phase, the ALHAT system generates an onboard Digital Elevation Model/Map (DEM) in real time of the local terrain, which is analyzed through sophisticated onboard Hazard Detection (HD) algorithms to determine safe landing sites, along with prominent terrain features. ${ }^{4}$ The ILP itself may or may not be a high-probability safe site within the region of the DEM, so the onboard GN\&C system assesses the ALHAT-provided safe sites and determines divert maneuvers to the desired safe location (i.e., the hazard avoidance part of HDA) based on a site safety ranking and fuel requirements.

Subsequent to the safe site determination, the ALHAT system implements HRN to track one of several prominent terrain features. The DEM generated by HD is stored onboard and used for comparison with additional images taken during HRN. Since no Nav system is perfect, there will be map-tie error associated with the DEM. The ALHAT Nav architecture implements a dual-state filter that blends inertial and targetrelative knowledge. In this manner, the map-tie error is not reconciled between the local DEM and the global maps used for TRN. During HDA and HRN phases up to landing, the only ALHAT objective is to ensure a safe landing within the local landing-precision requirement. To accomplish this task, ALHAT Nav processes HRN updates to minimize additional knowledge error growth after the time when the DEM was generated.

\section{Current Generation ALHAT Sensors}

The sensors within the current-generation ALHAT sensor suite include a long-range Laser Altimeter (LAlt), a Navigation Doppler Lidar (NDL), and a flash Lidar (FL) based Hazard Detection System (HDS). Images of the current-generation prototypes are shown in Figure 2. These sensors, along with the spacecraft Inertial Measurement Unit (IMU), star tracker, and other navigation sensors are processed within ALHAT Nav during the TRN, HD, and HRN phases of descent to provide precise knowledge for the vehicle GN\&C system to divert and land safely at the ALHAT-determined site. A notional timeline is shown below Figure 1 to indicate when various ALHAT or other onboard sensors are online and processed within ALHAT Nav.
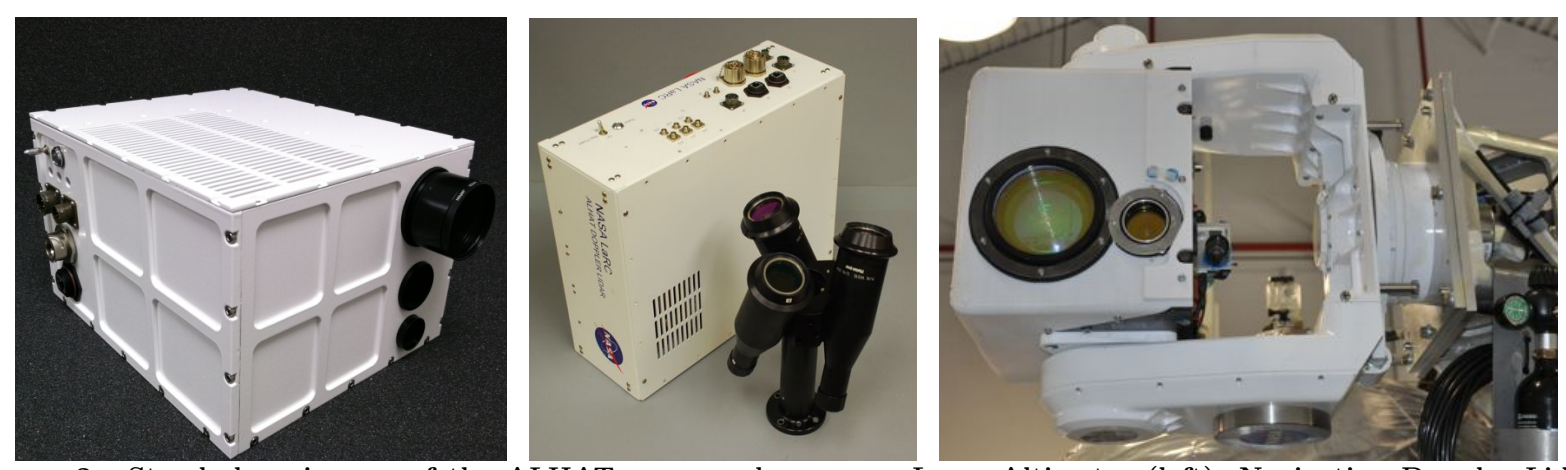

Figure 2. Stand-alone images of the ALHAT sensors: long-range Laser Altimeter (left), Navigation Doppler Lidar optics head and electronics box (center), and Hazard Detection System gimbaled flash lidar (right).

The current-generation LAlt developed through NASA LaRC is shown in Figure 2 (left). The LAlt is designed to provide high precision Line of Sight (LOS) range measurements that are used to conduct TRN from altitudes in excess of $20 \mathrm{~km},{ }^{2}$ as indicated in the timeline at the base of Figure 1 . The measured ranges to the surface are compared to onboard orbital-reconnaissance data to maintain minimal position error dispersions during descent. Currently, the performance objectives for the altimeter are met by using laser-based technology. The prototype used in the ALHAT sensor suite has an operational range that exceeds $30 \mathrm{~km}^{12}$ in Earth's atmosphere and is used to support surface-relative state estimation and navigation during ALHAT/Morpheus precision landing demonstrations since the Morpheus vehicle cannot fly to high enough altitudes or velocities to effectively demonstrate Lidar-based TRN. Range measurements are made with 
an advanced time-of-flight receiver capable of 2.5 -cm precision and with a dynamic range that exceeds 64 $\mathrm{km}$. The receiver incorporates a Constant False Alarm Rate (CFAR) system that maintains the false alarm rate below $2 \%$. This system also minimizes background noise contamination due to solar or plume thermal radiation. The transmitter laser operates at an eye-safe wavelength, which simplifies operations and testing on Earth, but future space qualified LAlt implementations will not require this feature, which will enable significant mass and volume reductions. Care and effort were placed on ruggedizing the enclosure and protecting the components to withstand the thermal and mechanical stresses experienced during terrestrial ALHAT test flights conducted aboard helicopters, fixed-wing aircraft, and Morpheus.

The current-generation NDL optic head and its electronics box are shown in Figure 2 (center). The NDL was developed through NASA-LARC and has been validated and revised throughout numerous ALHAT field test campaigns on airplanes and helicopters. ${ }^{15-17}$ These field tests have taken the NDL from demonstrations of the basic measurement concept with early prototypes, through hardware, electronics, and packaging revisions, and to implementation and performance characterization of the current-generation sensor. The current-generation NDL design is based on an all fiber-optic component architecture that is compact, robust, and simple to integrate into a host vehicle. The primary components of the NDL include the seed laser, fiber amplifier, and the transmitter/receiver optical telescopes. The laser wavelength is in the near infrared making the accuracy of the measurements orders of magnitude greater than conventional radar. The NDL telescopes transmit three collimated, diffraction-limited, 50-mm diameter beams, and the full angle divergence of each beam is approximately 31 micro-radians, which produces very small ground footprints and makes topographical effects negligible. The transmitter waveform is Frequency Modulation Continuous Wave $(\mathrm{FMCW})$, and all signals are captured in the frequency domain. This technique has several advantages over conventional pulse-pair processing Doppler Radar, ${ }^{18}$ including better accuracy and a smaller beam footprint. Additionally, velocity and range measurements can be obtained from all three beams simultaneously in realtime, thus providing velocity vectors, altitude, and 2-D ground relative attitude at the design data output rate of $20 \mathrm{~Hz}$. The current-generation NDL would provide measurements to ALHAT Nav from altitudes starting between 2-4 kilometers down to 30 meters, as indicated in the timeline at the base of Figure 1. The 30 meter altitude cutoff was intentionally chosen to avoid potential measurement corruption due to the lander plume interacting with the surface.

The current-generation HDS is shown in Figure 2 (right). The development and testing of the HDS was performed in collaboration between NASA JPL and NASA LaRC. The key to the successful HDS collaboration and joint development was the constant communication between the centers, the willingness to travel as required for integration efforts, and the development of detailed software and hardware interface specifications. NASA-LaRC was primarily responsible for development of the FL and its associated electronics and algorithms for real-time image generation, and NASA-JPL was primarily responsible for the overall HDS design (two-axis gimbal, dedicated IMU, and electronics) and the algorithms associated with generating the DEM and processing HD and HRN measurements in real time. The nominal operational range for the HDS, as indicated at the base of Figure 1, is for ranges of 1-kilometer to 100-meters on the Moon; the maximum slant range for flights on the Morpheus vehicle is approximately 460 meters. The current-generation HDS design was driven by landing-site requirements for an Altair-class human lunar lander; ALHAT considers these requirements on hazard detection and safe-site determination to be more stringent (and thus more conservative) than what would be required for a robotic lander. These requirements were to detect landing sites from a 1-km distance that are free of $30-\mathrm{cm}$ roughness hazards and surface slopes exceeding $5^{\circ}$. The HDS consists of a two-axis gimbaled FL, a dedicated IMU, custom processing electronics, and stand-alone power. The HDS contains its own closed-loop control system that makes use of the ALHAT Nav solution to determine gimbal slew angles and relative state information for taking images of the terrain surrounding the ILP. For the HDS to conduct DEM generation followed by HD and HRN in real time during decent, its electronics architecture makes use of hardware-based time stamping and parallel processing for the realtime sensor fusion and computation required to determine safe sites and to track features. ${ }^{19}$ Details on HDS-specific operation, including the HD and HRN phases, can be found within the references. ${ }^{20}$ 


\section{Preparation of Stand-Alone Sensors}

The three ALHAT sensors work together through ALHAT Nav to provide terrain-relative state measurements that enable autonomous and safe precision landing. The overall ALHAT system performance is tied to the individual performance of each stand-alone sensor. Each of the ALHAT sensors underwent stand-alone ground and flight tests to evolve the sensor designs and mature the algorithms, as well as detailed alignment and calibration testing. Details of past flight and development tests can be found within the numerous ALHAT field and flight test references. ${ }^{10,13-15,21,22}$ This section describes some of the stand-alone preparation and lessons learned for the current-generation ALHAT sensors leading up to flight tests on Morpheus.

Two copies of the HDS were constructed, which provided a backup version and enabled remote and local testing. Only the primary flight version of the HDS was fully integrated with the LaRC-provided FL and the JPL-provided gimbal and interface hardware. This version, along with the second FL and its electronics remained at LaRC for development and calibration prior to integration and testing on Morpheus. The second HDS gimbal and the remaining HDS electronics remained at JPL for development and testing. This second version uses an eye safe Advanced Scientific Concepts, Inc. (ASC) TigerEye flash Lidar; ${ }^{23}$ the TigerEye camera is also used within the HDS FL. Development testing at JPL was conducted in a dedicated lab (Figure 3, left), and development testing at LaRC was conducted within several lab and hangar facilities, as well as on the LaRC Long Distance Test Range (LDTR) (Figure 3, right).
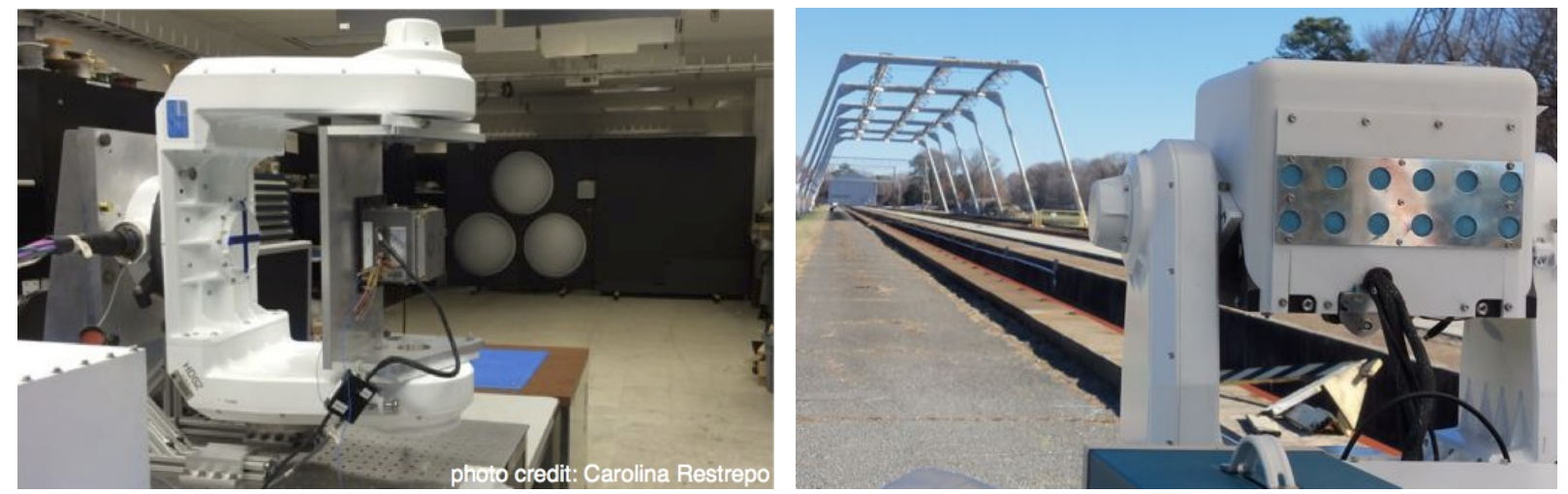

Figure 3. A JPL lab (left) was used to test pointing, HD and DEM-generation algorithms with a surrogate, eye-safe FL, and the LaRC LDTR (right) was used for integrated-HDS testing, including calibration of the non-eye-safe HDS FL and the overall HDS performance.

The JPL development lab includes an HDS mounted to an optic bench and several target boards with mounted hazards; the side mount configuration for the HDS was added during flight testing on Morpheus so that flight test analyses could be performed in the flight test configuration. The ability to develop and test in tandem greatly aids the development and maturation of both the hardware system and the algorithms. Additionally, the lab setup allows the replay of flight test data (however, without dynamics) to further assess and refine HDS algorithms and operation. Since the HDS is a stand-alone closed-loop control system that requires per-axis pointing control within $0.2^{\circ}$ and per-axis pointing knowledge within $0.15^{\circ},{ }^{20}$ tight intercomponent time stamping and alignment knowledge are critical for operation. During early development, internal interfacing and timing between the gimbal, IMU, and FL were conducted in the lab with real-time testing to confirm performance. For precision alignment, built-in fiducials were used to define component-tocomponent alignment datums that were measured with metrology systems to establish alignment transforms. Imaging the target boards to generate a DEM and perform HD and HRN helped mature the algorithms and enabled testing and verification of computational efficiency improvements.

The LaRC LDTR has a range in excess of 800 meters and a target wall with mounted hazards; the wall is visible in the background of Figure 3 (right). The facility was used for both range accuracy and range precision calibrations of the stand-alone FL, as well as static and dynamic pointing and algorithm performance testing for the integrated HDS. The facility was surveyed in detail with both GPS longduration dwells and theodolite measurements to establish target and reference locations. The HDS testing 
then established relative alignment with metrology measurements between the HDS datums and the LDTR reference locations. For the FL inter-image accuracy calibration, the sensor was mounted to a static optic bench on the LDTR, and targets were placed at precise ranges. For the FL intra-image pixel-to-pixel precision calibration, the FL was mounted to an optic bench inside an instrumented Lidar track and it imaged the flat wall of the LDTR target building over expected flight ranges. The FL inter-image range accuracy was calibrated to within $20-\mathrm{cm}(1 \sigma)$, and the intra-image pixel-to-pixel precision was calibrated to within 8-cm $(1 \sigma)$ for the planned Morpheus ranges. Metrology and optical alignment methods were also used to identify to high precision the FL boresight vector relative to the HDS gimbal axes. With knowledge of the boresight vector, a detailed pointing and alignment test was conducted within a hangar at LaRC and at the LDTR to demonstrate superior HDS pointing knowledge to within $0.05^{\circ} .{ }^{20}$ Establishing HDS pointing knowledge well within requirements provided margin on the required Nav pointing knowledge of $0.1^{\circ}$ per axis for imaging terrain for DEM generation and HRN measurements.

The development and testing of the NDL and LAlt sensors were conducted at NASA-LaRC. Two copies of the LAlt and NDL were constructed to have a backup version and to enable simultaneous remote and local testing and analysis with the sensors on Morpheus and the sensors at LaRC. The development and performance verifications of the NDL and LAlt were conducted between lab tests and field tests. The current-generation NDL includes three beams that point $22.5^{\circ}$ from nadir and are spread at $120^{\circ}$ heading increments. Development and testing of the NDL electronics required stimulation of the detector for each of the beams, so the lab environment used treadmills and belt sanders to generate measurement signals. These tests were not used for measurement calibration but were instead used for testing and revision of the NDL electronics, system timing, data acquisition and processing. To establish the NDL LOS velocity accuracy for each beam, measurements were taken of the motion of a high-fidelity and very-low-distortion subwoofer driven at low frequency. Comparisons of the speaker surface velocity (independently measured) to the NDL beam LOS-velocity measurements indicate sub 1-mm/sec accuracies; the accuracy determination was limited by the NDL signal-processing quantization. To verify real-time autonomous signal acquisition and processing, dynamic swing tests of the NDL were conducted on a NASA-LaRC gantry (Figure 4).
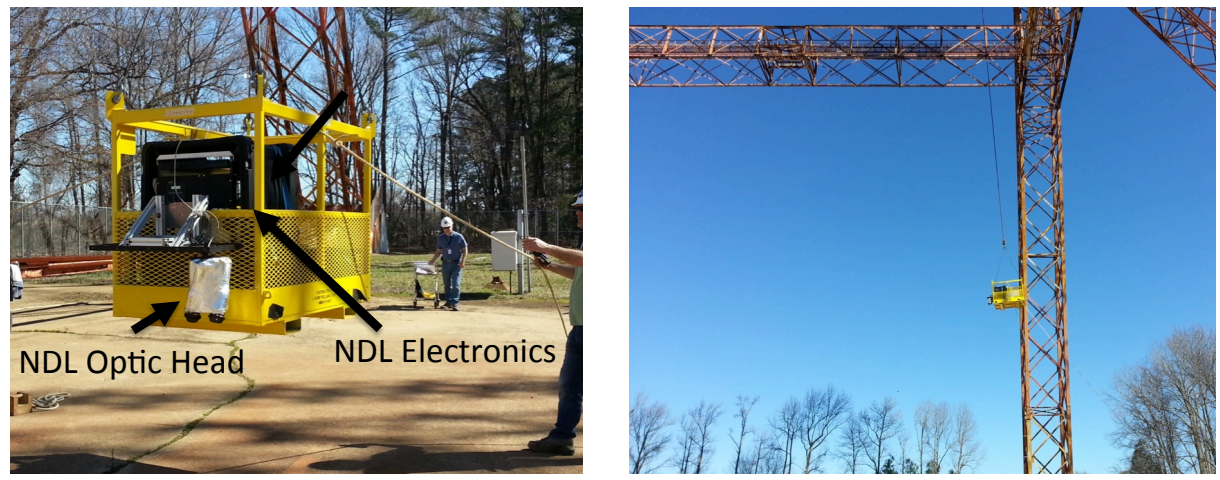

Figure 4. Gantry swing test at LaRC with Doppler Lidar optic head and electronics.

During the terrestrial flight testing prior to Morpheus, the three-beam NDL measurements were compared with both Global Positioning System (GPS) derived measurements, as well as high precision IMU-based measurements made with an Applanix inertial navigation system, which showed vector velocity component agreement between the Applanix and NDL to better than $3 \mathrm{~cm} / \mathrm{s} .{ }^{17}$ Primary error sources within the NDL measurements include wavelength accuracy of the source laser, attitude rates of the sensor and HV, and pointing knowledge for each of the three laser beams. An overview of the NDL development, including an error analysis of the measurements, is provided within a prior reference. ${ }^{14}$ The wavelength was calibrated within $10^{-7}$ of the desired wavelength, which ensures that wavelength error is negligible. The attitude rates of the Morpheus vehicle are well below the rotation rates at which NDL measurement errors are significant. To reduce error associated with pointing knowledge, metrology measurements were taken for each beam over a 23 meter path length to define beam direction relative to a hardware-established NDL sensor frame. 


\section{Simulations of Integrated ALHAT and VTB Systems}

The ALHAT project extensively used simulation tools throughout development of the sensors and the algorithms, as well as for the planning of flight test activities. Early ALHAT simulations provided necessary performance metrics for both designing and revising the sensor designs and specifications that drove the implementation of the current-generation ALHAT sensors. Additionally, these simulations provided insight into algorithm performance and parameter sensitivities within the sensors. The integrated ALHAT/Morpheus 6-DOF Simulation (Sim) was developed to provide GN\&C insight into open- and closed-loop performance with ALHAT sensors operating in the expected Morpheus flight-test regime, which aided in the refinement of flight profiles for properly exercising the ALHAT sensors in a lunar-like approach trajectory. Additionally, these simulations provided the ALHAT team with an integrated-system understanding of the Morpheus vehicle dynamics that were used within sensor-specific, high-fidelity simulations to refine and tune algorithm parameters and sensor operation for the anticipated flight profiles. The simulations do not replace the results from actual flight testing, so actual flight-test data is continually analyzed and used for refinement of the simulations, helping to improve the overall tool set for predicting, planning, executing and analyzing.

The development of the joint Morpheus/ALHAT Sim was performed with the NASA-JSC Trick simulation tool for simulating 6-DOF dynamics. The Sim runs the actual Morpheus Flight Software (FSW) that is executed onboard the vehicle during flight tests. To make Morpheus a viable, autonomous VTB for testing new, prototype GN\&C technology, a dual-string Navigation capability was implemented within the GN\&C FSW architecture. The Sim captures this capability, as illustrated to high level in Figure 5. This architecture maintains a core VTB Nav capability that the Autonomous Flight Manager (AFM) uses to monitor ALHAT Nav performance to enable onboard testing and faster flight-to-flight refinement of ALHAT Nav and ALHAT sensors without jeopardizing vehicle safety. As ALHAT (or any other prototype GN\&C system) matures, flight tests can progress from initial open-loop flights to full closed-loop flights with VTB Nav as a backup in case the prototype GN\&C system performs outside of specified tolerances. This capability (both onboard and in the simulation) allows Morpheus to accelerate the development and maturation of new NASA GN\&C technologies for flight applications.

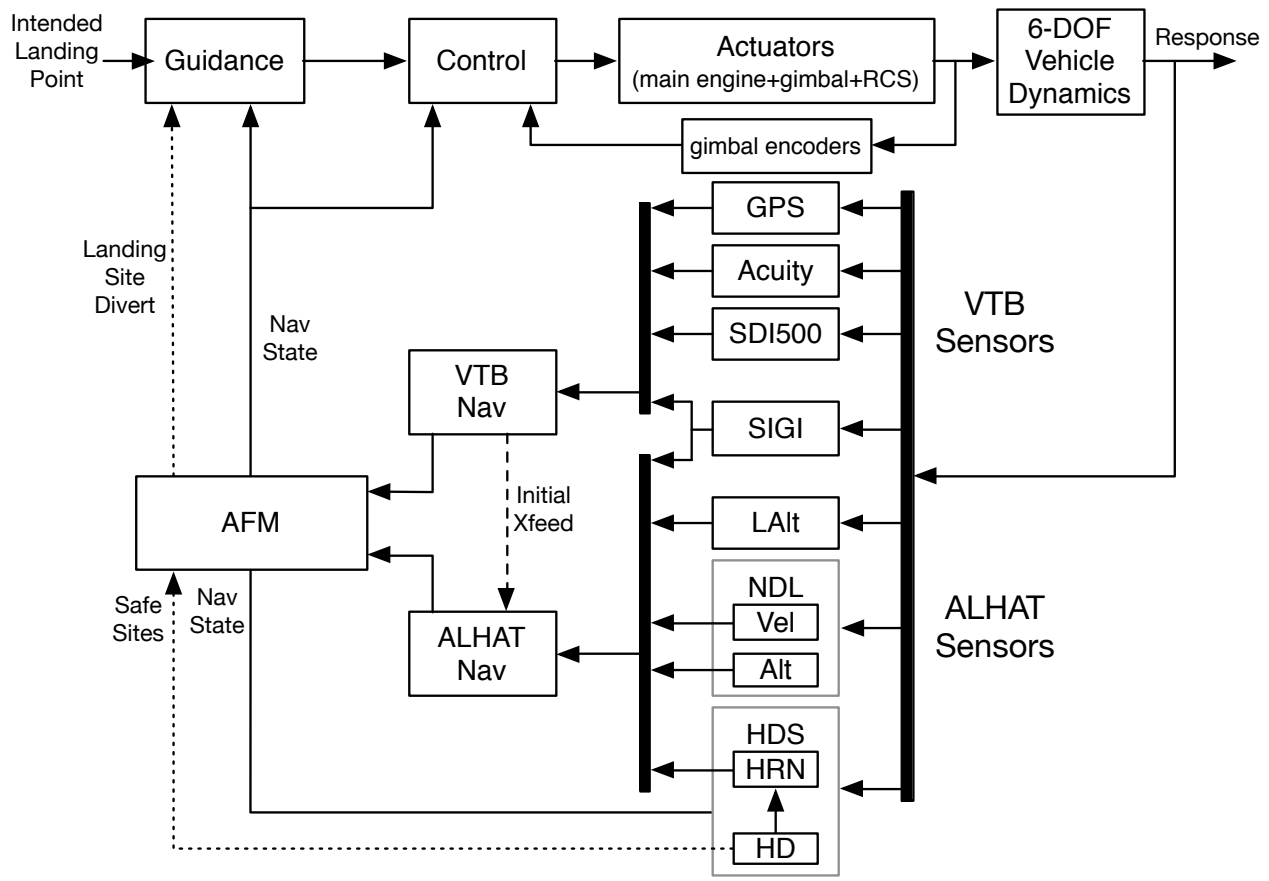

Figure 5. High level diagram of the 6-DOF simulation environment for testing and analyzing ALHAT versus VTB GN\&C flight software performance. 
The Sim illustration in Figure 5 summarizes the interaction between the ALHAT and VTB sensors with the dual-string Nav filters (VTB and ALHAT) and the GN\&C FSW on Morpheus. The diagram simplifies the full FSW implementation but provides the basic interaction between components. The AFM provides oversight during the flight, including sequencing of the sensors being processed in the Nav filters, specifying the Nav state utilized within Guidance and for HDS pointing, and determining the landing site used for the HDA divert (i.e., ALHAT selected site or surveyed truth site). The Sim includes high-fidelity models for the VTB sensors: Global Positioning System (GPS), Acuity distance measurement sensor (AR3000), gimbal position encoders, Space Integrated GPS/INS (SIGI) used as the primary IMU, and Systron Donner IMU (SDI500) used as the backup IMU. Both ALHAT Nav and VTB Nav share a common vehicle IMU, as indicated in Figure 5. The Sim also includes high-fidelity models of the ALHAT LAlt and NDL sensors, along with a medium-fidelity model of the HDS functionality.

Models for the NDL and LAlt include high fidelity algorithms that account for the effects of laser speckle, signal to noise ratio, and vehicle dynamics on measurement accuracy and precision, as well as quantization and noise uncertainties relevant to the Morpheus flight regime. The NDL model also includes dynamics effects due to translational and rotational motion. These models were verified against stand-alone ALHAT sensor models that were developed and refined in ALHAT field testing prior to integration with Morpheus. The NDL model, in particular, is useful for analyzing sensitivities and expected accuracies of ALHAT Nav due to variations in the NDL measurements, such as misalignment errors in beam directions or beam obscuration during flight (e.g., dust, plume, etc.).

The Sim implements only a medium-fidelity model of the HDS measurements. Since the HDS is a complex, closed-loop control system with multiple sensors and an actuated gimbal, a high-fidelity model would add significant computational overhead and modeling details unnecessary for the integrated simulation. The medium-fidelity model captures the uncertainty effects, as well as the knowledge tolerances affecting the HDS measurements. The non-dynamic, medium-fidelity model was determined to be adequate for the Sim based on physical tether testing of the integrated ALHAT/Morpheus system. Tether testing showed no significant GN\&C response to HDS motion during HDS DEM generation and HRN pointing operations. The HDS model includes errors sources coming from internal HDS pointing and FL range knowledge, as well as the external ALHAT Nav solution used for targeting terrain locations for HDS imaging operations. Additionally, the Field of View (FOV) boundaries for the FL optics are included since significant ALHAT Nav errors can cause tracked HRN features or safe sites to be outside of actual imaged terrain. The illustration in Figure 6 shows how the HDS DEM can be offset from the initial desired location due to error in both ALHAT Nav and the HDS pointing/range knowledge. ALHAT Nav estimates the location of the ILP (where the DEM is centered), so errors in ALHAT Nav cause an offset. Additionally, since the HDS does not have perfect pointing knowledge or a perfect FL range, the images used to assemble the DEM will be offset from truth, as will be the HDS computed safe site (SS1 in the illustration) from the surveyed safe site (LS1). These same error sources also affect HRN, which is not shown.
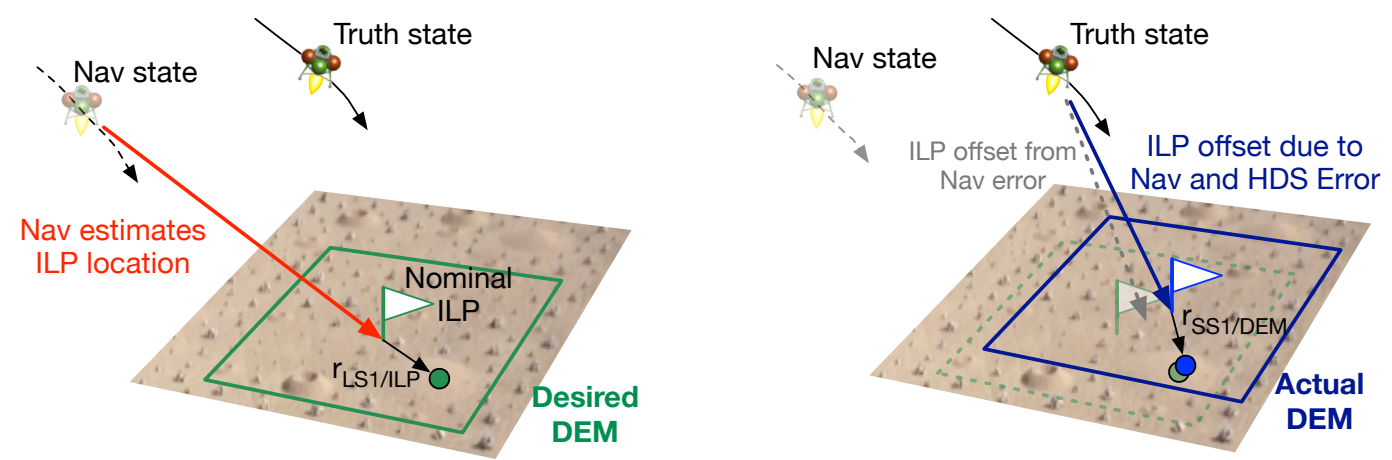

Figure 6. The simulation contains detailed models of all ALHAT sensors. Pictured here is a simplified illustration of the DEM generation and safe site determination that depicts offsets due to Nav and HDS errors. These offsets also impact HRN measurements (not illustrated).

A stand-alone high-fidelity simulation for the HDS was developed at NASA-JPL for running Monte 
Carlo analyses with Morpheus Sim trajectories and HDS flight software. This stand-alone tool enabled the ALHAT team to fine tune HDS DEM-generation, HD and HRN parameters, as well as gimbal pointing control parameters to improve HDS operations in flight. Additionally, the flight trajectories could be replayed through an HDS Hardware-in-the-Loop (HWIL) simulations at JPL to command gimbal motion (albeit without vehicle dynamics) through DEM generation mosaics and HRN pointing to fine tune the gimbal control system parameters and to refine the systems-level knowledge of the time required for DEM generation and HRN measurement processing. These HWIL simulations were performed in the NASA-JPL lab shown in Figure 3, which uses the backup HDS. The HWIL sim can reprocess flight FL images, synthetic images, or images from the surrogate HDS FL images depending on the objective of the lab test.

\section{Test Environment Preparations}

The environmental considerations for the ALHAT/Morpheus flight campaign include the vehicle-induced flight environment and the local ground and atmospheric environment in which the sensors operate. The vehicle-induced flight environment includes structural vibration, plume-induced thermal effects and takeoff/landing debris that affects sensor hardware. The ground environment includes the physical terrain layout and its material properties, such as state (solid or liquid) and reflectivity. The atmospheric environment includes local humidity, aerosols and other particulates during flight tests.

The current-generation ALHAT sensors were put through both thermal and vibration testing in early 2012 (Figure 7). The thermal profiles were based on early model-based assessments and tether flights of the first Morpheus vehicle. The vibration spectrum was based on a NASA Workmanship spectrum that was considered sufficient and of appropriate magnitudes for the eventual Morpheus free flights. This testing led to minor revisions within the ALHAT electronics to secure components from damage due to expected flight vibration levels. Additionally, some modifications were made to electronics components to ensure operational robustness at the expected operating temperatures.
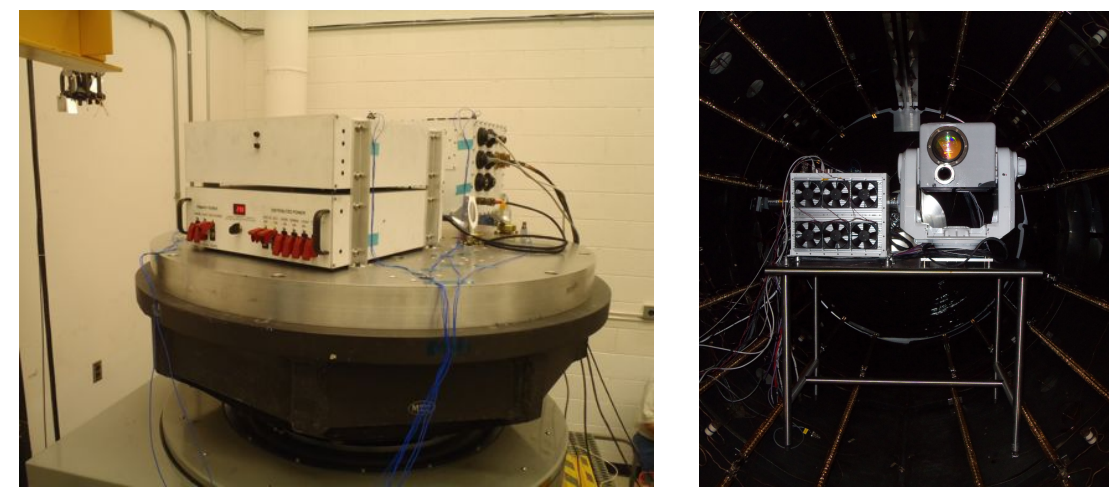

Figure 7. Environmental testing of ALHAT sensors and electronics was performed to workmanship vibe spectra (left) and to thermal conditions anticipated for flight testing onboard Morpheus (right).

The terrain environment for Morpheus/ALHAT flight testing was developed along the NASA-KSC Shuttle Landing Facility (SLF). This site was chosen because of the extensive length of smooth runway and relativelysmooth terrain, along with the wide region of undeveloped land at the northern end that provided safety zones for the tests. The SLF is surrounded by a narrow waterway, so trajectory simulations were used to confirm that ALHAT sensor placement would not point the lasers at the water, which causes measurement dropouts. Land near the northwest corner of the SLF runway was tailored to resemble the distribution of rocks and craters within a smooth mare region of the Moon. A detailed lunar DEM (Figure 8, left) was developed from actual Moon imagery and used to guide the design and construction of the ALHAT lunar-like hazard field (Figure 8,right). During Morpheus/ALHAT flights, the HDS images the hazard field to find safe sites during HDA and for feature tracking during HRN.

The hazard field was constructed with broken concrete debris from prior construction projects, plus crawler fines left over from the Space Shuttle program. The crawler fines are remnants of gravel crushed 

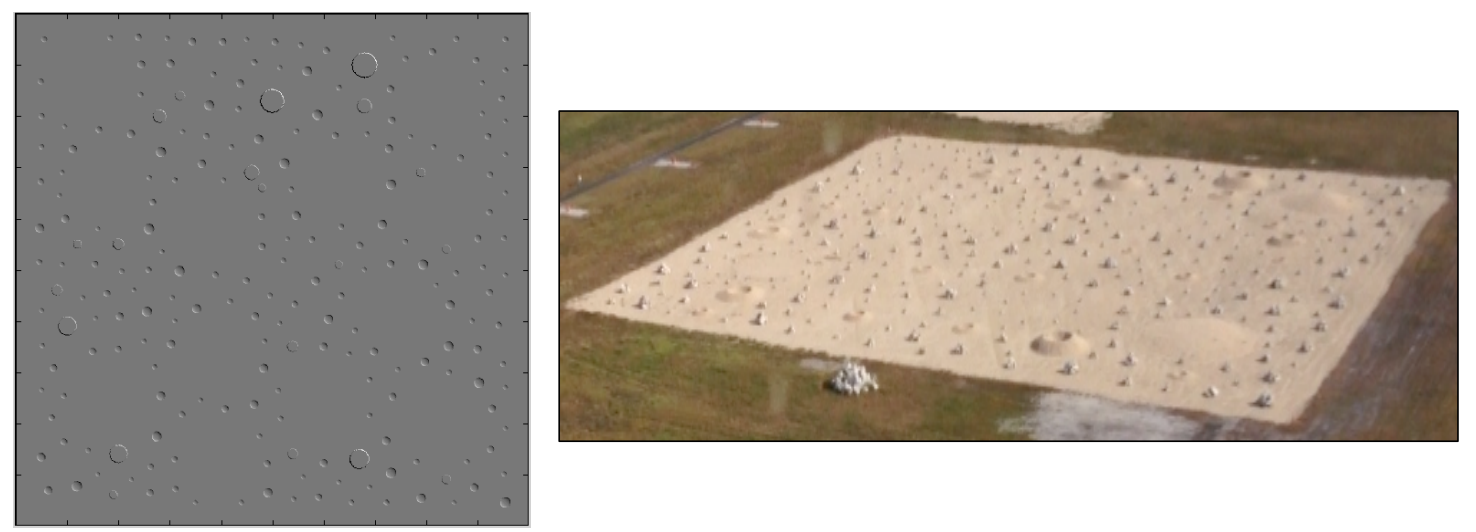

Figure 8. Lunar terrain model (left) used in the development of the ALHAT hazard field (right) at KSC. The craters in model are clearly visible in the constructed field.

under the weight of the crawler, mobile launch pad, and orbiter as the assembly moved between the Vehicle Assembly Building (VAB) and the launch pad. The hazard field contains large and small rocks, as well as several sizes of craters as seen in the right-side image. Additionally, many relatively flat regions are visible. Two of the larger flat regions are actually concrete pads that were covered with crawler fines to ensure uniform surface reflectivity for HDS FL imaging. The concrete debris and the crawler fines were evaluated by NASA-LaRC to verify that their reflectivities were within the dynamic range of the FL and to tune the FL Auto Gain Control (AGC) algorithm.

The current-generation ALHAT sensors are designed for operation within limited or no atmosphere, so terrestrially flight testing ALHAT on Morpheus adds further environmental considerations in the preparation of the sensors. The presence of wind-borne particles or other aerosols within the atmosphere add noise or attenuation to the sensor measurements. Some of these obstructions are caused by the vehicle interacting with the environment - kicking up dust or debris on takeoff or landing, or the wind blowing plume-heated air directly into the sensor LOS. Completely eliminating these error sources is not possible, but they are not a show stopper to flight testing. The ALHAT Nav algorithms can handle intermittent drops in NDL or LAlt measurements and still maintain a precise knowledge state. In contrast, the HDS requires fairly clean FL images for generating the DEM to determine safe sites and making HRN measurements. The environmental preparations to handle airborne disturbances primarily included wind placards to minimize particulates and heated air from obscuring the FL images. Additionally, debris baffles were added to the NDL and LAlt, as seen in Figure 9, to minimize the potential for direct impacts to the lenses on takeoff and landing.
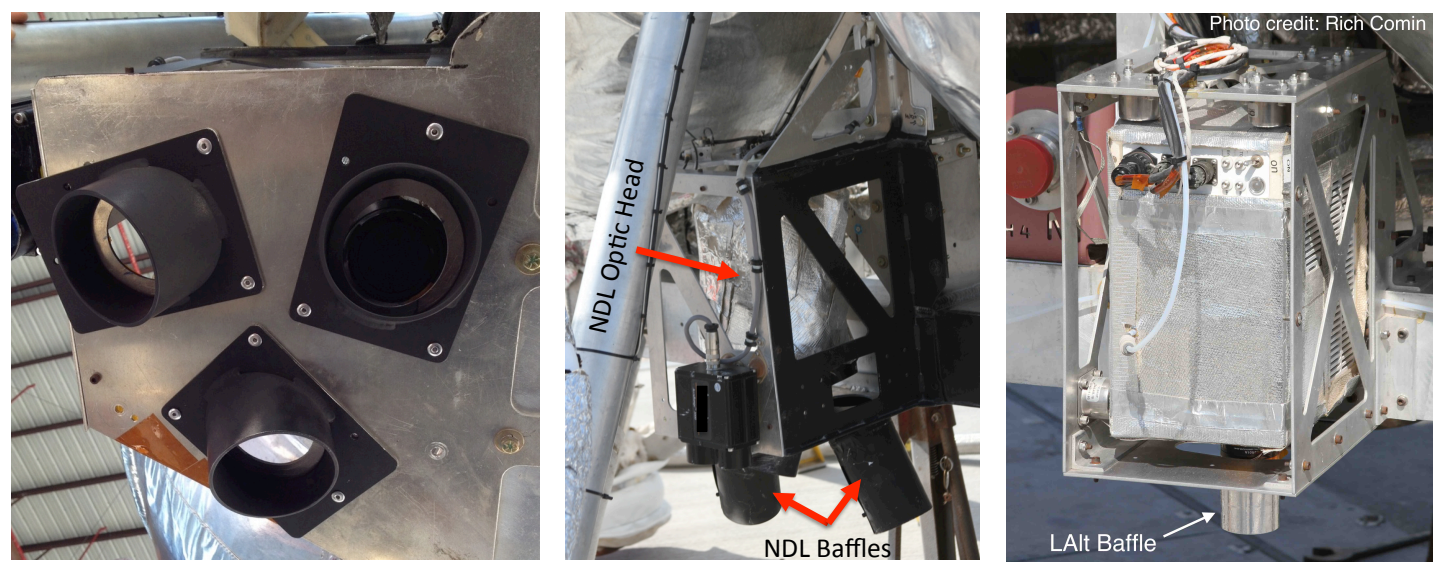

Figure 9. Baffles protect NDL and LAlt scopes from takeoff and landing ground ejecta; view from base of NDL optic head without baffle sleeves (left), side view of DL with full baffles attached (center), and view of LAlt with baffle (right). 
The humidity during each flight day also became a concern for HDS operation as the current-generation FL camera is not hermetically sealed. A small dew point spread from ambient temperature can damage the FL detector pixels, which adversely impacts the DEM and HRN images. To mitigate humidity concerns, a dew point spread requirement was used to dictate whether or not purge air was needed. A continuous dry-air purge was used on the FL during all non-flight days and up until vehicle roll-out on flight days. When the vehicle was rolled out for flight, a portable carbon-dioxide $\left(\mathrm{CO}_{2}\right)$ bottle was attached to the vehicle to provide purge during transit from the hangar to the pad, followed by a gaseous nitrogen $\left(\mathrm{GN}_{2}\right)$ purge on the pad up until final ground-personnel pullback prior to flight. The $\mathrm{CO}_{2}$ purge was put back in place for transit from the landing pad back to the hangar following flight tests.

\section{Integration and Functional Testing between ALHAT and VTB}

Integration and testing between the ALHAT and Morpheus electronics began well before the Spring 2014 flight testing. In the Spring of 2012, ALHAT conducted dynamic tests of the ALHAT sensors on a truck driving at the LaRC-LDTR facility (Figure 10).$^{20}$ During initial truck testing, Morpheus avionics and sensors were not available for interfacing with the ALHAT sensors, so a Charles Stark Draper Laboratory (CSDL) Guidance Embedded Navigator Integration Environment (GENIE) ${ }^{24}$ system provided the required Nav solution for HDS pointing to the LDTR target building for HD imaging. Once Morpheus sensors and avionics became available, they were used in later truck testing. These initial tests provided early interface and operations opportunities between the ALHAT and Morpheus projects. In addition, ALHAT conducted helicopter flight testing with Morpheus avionics in late 2012 at the NASA-KSC SLF to obtain dynamic flight data for maturation and preparation of the current-generation ALHAT sensors prior to re-integration and free-flight testing on the Morpheus vehicle. ${ }^{13-15}$
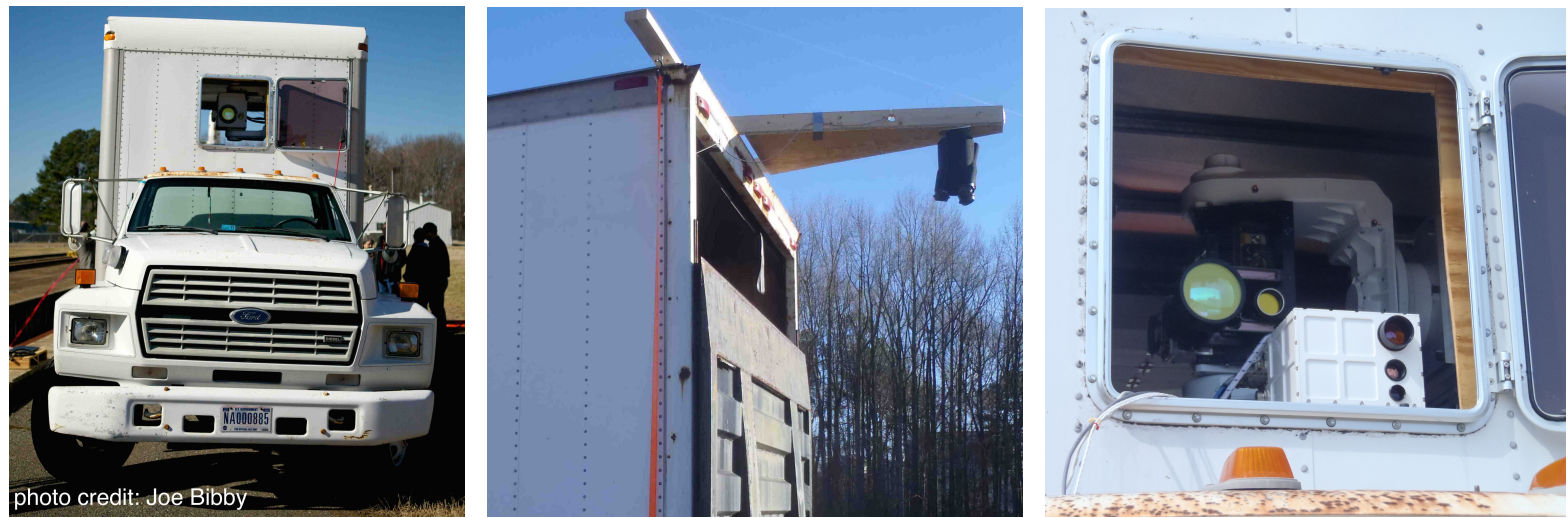

Figure 10. ALHAT conducted dynamic testing on a truck (left image) at the NASA-LaRC LDTR. The NDL was mounted to the aft roof of the cargo area (center image), and the HDS and LAlt were mounted inside of the cargo area with a forward view through an access window (right image).

The command and telemetry interfacing between the ALHAT sensors and the Morpheus avionics was accomplished through a series for bench top tests (Figure 11, left), also referred to as FlatSat tests (implying essentially the high-fidelity interfacing of satellite or spacecraft electronics and sensors on a flat benchtop). The FlatSat testing was critical for ironing out software interfaces between the ALHAT and HV systems, as well as the command functionality, telemetry, and time synchronization. The FlatSat testing included commanding of all HDS operational modes to verify correct Nav state inputs and HDS responses per the interface design. These tests were conducted without actual imaging because the HDS FL is not eye safe and is defocused at short ranges. The LAlt and NDL were also tested in this manner, with the NDL detector stimulated with a belt sander to provide measurement flow through the telemetry system. One other focus of FlatSat testing was the verification of Nav state initialization and drift. Due to the confined region of the ALHAT Hazard Field for ALHAT precision landing, and especially for HDS scanning, a stand-alone Nav Testbed (NavTB) was developed to assess attitude and position of the VTB Nav state (Figure 11, 
right), which is also used for the state initialization of ALHAT Nav. The NavTB consisted of a precisely surveyed (position and attitude) test structure with alignment features for VTB Nav sensors. The NavTB provided an independent knowledge state for comparison of VTB Nav initialization and drift; the VTB sensors could be removed after initialization, driven around the test site, and then precisely realigned to the NavTB structure to assess drift. This was an extremely rapid and low cost method to perform a partial, independent verification of the Nav state.
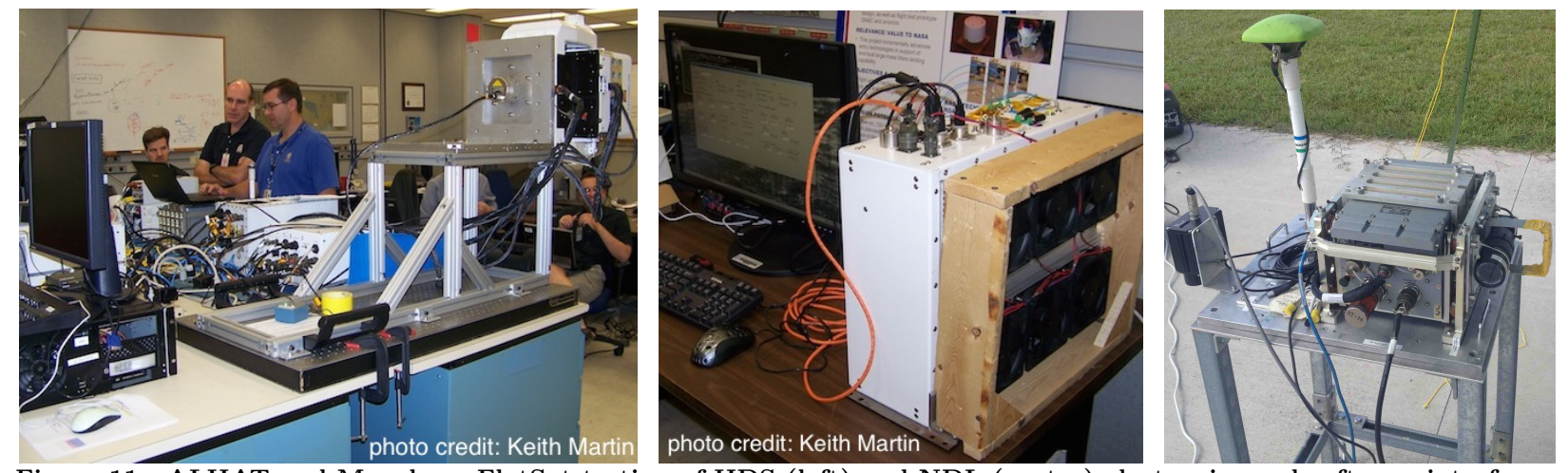

Figure 11. ALHAT and Morpheus FlatSat testing of HDS (left) and NDL (center) electronics and software interfaces, plus the NavTB for assessing Nav pose initialization and drift (right).

Electrical and mechanical integration of the ALHAT sensors onboard Morpheus made use of well-defined electrical and hardware interface descriptions, formally documented using Interface Control Documents (ICDs). The electrical ICDs define the wire harness interconnects and the overall grounding and shielding scheme. The hardware ICDs define the mechanical provisions for mounting and properly securing the ALHAT sensors onto the vehicle. In addition, the hardware interfaces include alignment features that are essential for translating sensor measurements into precise vehicle poses. On the sensor side, these alignment features define a reference frame for the ALHAT measurement alignment and calibration. On the vehicle side, these same features provide metrology fiducials for obtaining precise alignment position and orientation of the ALHAT sensors relative to the ALHAT Nav reference frame on the Morpheus structure. Photos of the hardware installation of several ALHAT components are shown in Figure 12.
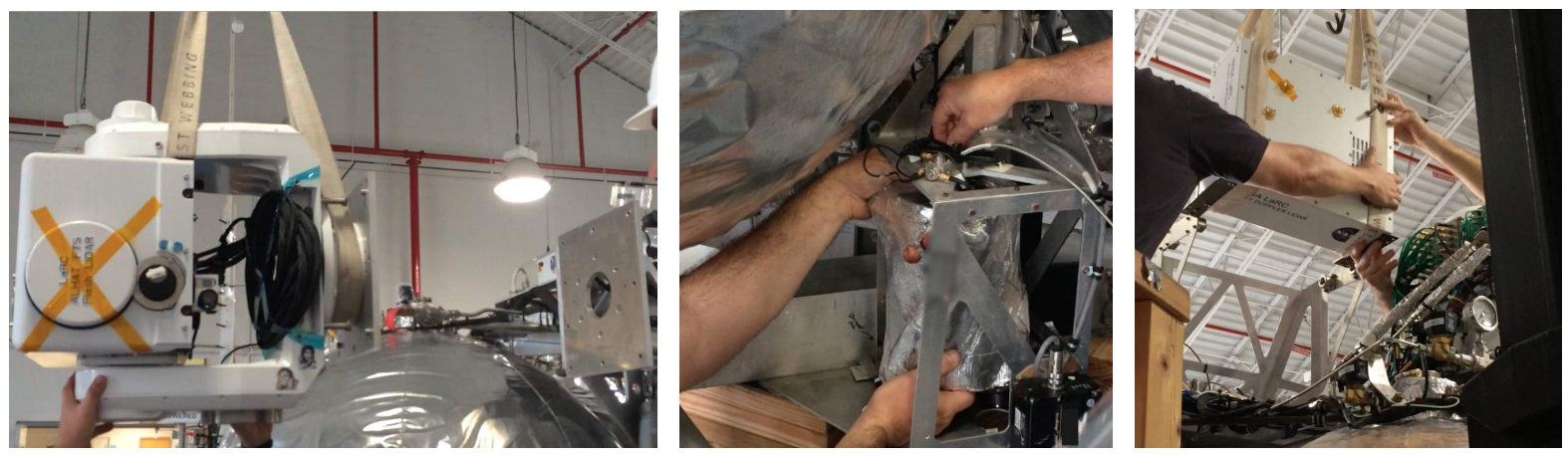

Figure 12. Installation of ALHAT components onto the Morpheus vehicle.

The integration and final functional verifications of the ALHAT sensors was accomplished within one week in early Spring 2014. The timely integration and verification in preparation for flight testing was primarily the result of the focused early integration testing in the FlatSat and ALHAT truck and helicopter tests, as well as prior tether testing with the first and second Morpheus vehicles. A key within all of this work was the constant lines of communication that remained open within the multi-center ALHAT and NASA teams, along with the development of clear ICDs for the hardware, electronics, software and power. Following the integration and preparation campaigns, the ALHAT sensors onboard the Morpheus vehicle were poised for 
free flight testing at the KSC SLF hazard field. Figure 13 provides an illustration of the integrated locations of the ALHAT systems onboard Morpheus for the flight testing.
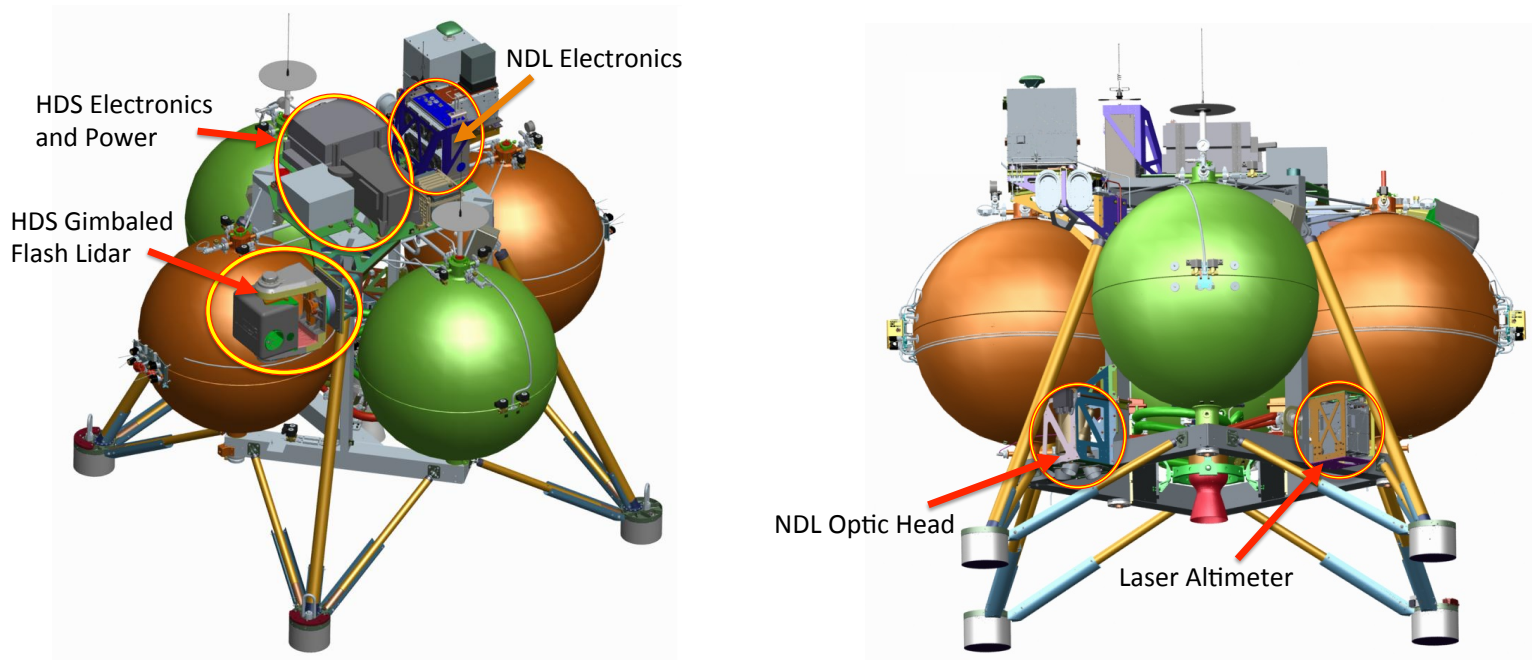

Figure 13. Illustrations of ALHAT sensors and electronics onboard the Morpheus 1.5B vehicle.

\section{Final Remarks}

The focus of this paper has been on the extensive work within the ALHAT and Morpheus teams to prepare the stand-alone ALHAT sensors for integration and testing onboard the Morpheus vehicle. The integration and functional testing of the ALHAT sensors onboard Morpheus went efficiently and smoothly due to the rigorous planning and collaborative work between the teams over the past several years. The preparation and integration process has provided many lessons learned that have been mentioned throughout this paper. These closing remarks will reiterate some of the lessons learned that have made this undertaking both a highly-successful flight-test demonstration of new NASA autonomous precision landing capabilities and a highly-successful demonstration of multi-center collaboration within NASA.

Communication is key to successful collaboration and joint development between centers. In addition to regular teleconferences and one-on-one communications, the use of software, hardware, electronics and power ICDs and specifications greatly aided understanding and reworking differences in interconnections. Significant stand-alone development at team members' local centers was enabled through these ICDs, which sped up and reduced the amount of required travel time for deeper integration and testing.

The ability to develop and test in tandem greatly aids the maturation of hardware, electronics and software implementations. Further, the ability to simultaneously test between local and remote facilities speeds the debugging and refinement of systems. This follows the mantra of "test as you fly and fly as you test". These capabilities benefited not only analysis and refinement of the fully integrated systems but also the early development of each component. Being able to test new algorithms or electronics designs within breadboard and bench-top setups of the processing architecture provides an invaluable capability for rapid development and revision of a prototype system. For ALHAT and Morpheus testing, when possible, two versions of each ALHAT sensor and each Morpheus avionics component were produced. This not only provided backup systems but also allowed for simultaneous remote testing of the integrated systems alongside focused stand-alone testing at the local centers. In addition, testing stand-alone sensor hardware such as the HDS in the planned flight configuration aids in understanding expected flight performance and allows for more flight-like replays (without vehicle dynamics) of flight data to fine tune sensors and algorithms for future flight tests.

Timing is key to successful GN\&C sensor integration and operation. The ALHAT and Morpheus teams discovered very early through the closed-loop function of the ALHAT HDS that tight timing was critical 
for pointing and imaging operations during HDA and HRN. Significant efforts were put into designing and implementing the ALHAT sensors timing architectures, as well as the timing interfaces with the Morpheus vehicle, and yet timing remained one of the largest challenges to work through during integration and testing. Timing interfaces and time stamping warrant attention at all phases of development, implementation and integration and will be a continued focus area in future path-to-spaceflight ALHAT implementations.

Alignment and calibration is critical for precision landing GN\&C. The top-level requirements for ALHAT precision landing place very precise pose knowledge requirements on the measurements from the ALHAT sensors, as well as the knowledge state from ALHAT Nav (used for both vehicle GN\&C and for HDS closedloop pointing). The ALHAT team conducted extensive stand-alone sensor calibrations, plus used laser metrology to very precisely determine alignment within the sensors. Metrology was also used for integration of the sensors onto the vehicle to minimize knowledge error between the measurements and the ALHAT Nav frames.

Simulations provide insight into algorithm performance and parameter sensitivities within the sensors and the vehicle GN\&C system, although they are not a replacement for actual flight testing. Integrated ALHAT/Morpheus simulations and analyses provided insight into anticipated ALHAT performance within the Morpheus flight-test environment. These studies were used for parameter and algorithms refinements for each flight. No simulations identically capture flight performance however, so actual flight data was also used to fine tune the simulations, as well as refine ALHAT sensor and GN\&C system tunings.

The dual-string Nav capabilities of the Morpheus VTB provides a valuable capability for progressively and rapidly maturing the ALHAT system (or any other prototype GN\&C system). Flight tests were able to quickly advance from initial open-loop flights to fully closed-loop flights because the nominal VTB Nav always acts as a safety monitor and backup to takeover in the event of issues with the second, prototype Nav system. This capability allows Morpheus to accelerate the development and maturation of ALHAT toward future spaceflight applications.

\section{Acknowledgments}

We would like to acknowledge the hard work and dedication of all ALHAT and Morpheus team members, within many NASA centers and contractor organizations, who have together supported and made our flight testing possible. We would also like to acknowledge the ground support and operations groups at KSC who provided the facilities, logistical support and flight test coordination throughout the ALHAT and Morpheus flight campaigns. The work described herein was performed within NASA at the Johnson Space Center, Jet Propulsion Laboratory, Langley Research Center, and Kennedy Space Center as a part of the ALHAT and Morpheus projects. The ALHAT work at the Jet Propulsion Laboratory, California Institute of Technology, was performed under contract with the National Aeronautics and Space Administration (Government sponsorship acknowledged).

\section{References}

${ }^{1}$ Epp, C. and Smith, T., "The Autonomous Precision Landing and Hazard Detection and Avoidance Technology (ALHAT)," Proc. Space Technology and Applications International Forum (STAIF), 2007.

${ }^{2}$ Epp, C., Robertson, E., and Brady, T., "Autonomous Landing and Hazard Avoidance Technology (ALHAT)," Proc. IEEE Aerospace Conference (AEROCONF 2008), March 2008.

${ }^{3}$ Brady, T., Schwartz, J., and Tillier, C., "System Architecture and Operational Concept for an Autonomous Precision Lunar Landing System," AAS 30th Rocky Mountain Guidance and Control Conference, February 2007.

${ }^{4}$ Ivanov, T., Huertas, A., and Carson, J., "Probabilistic Hazard Detection for Autonomous Safe Landing," Proc. AIAA Guidance, Navigation, and Control Conference, August 2013.

${ }^{5}$ Johnson, A. and Montgomery, J., "An Overview of Terrain Relative Navigation for Precise Lunar Landing," IEEE Aerospace Conference (AEROCONF 2008), March 2008.

${ }^{6}$ Johnson, A. and Ivanov, T., "Analysis and Testing of a LIDAR-Based Approach to Terrain Relative Navigation for Precise Lunar Landing," Proc. AIAA Guidance, Navigation, and Control Conference, August 2011.

${ }^{7}$ McGee, T. G., Shankar, U., Shapiro, S., Shyong, W., Krupiarz, C., Reid, D., and Kaidy, J., "Performance Analysis of the 
APLNav System for Passive Optical Lunar Navigation," Proc. AIAA Guidance, Navigation, and Control Conference, August 2009.

${ }^{8}$ White, M., Criss, T., and Adams, D., "APLNav Terrain Relative Navigation Helicopter Field Testing," Proc. AIAA Guidance, Navigation, and Control Conference, August 2009.

${ }^{9}$ Amzajerdian, F., Pierrottet, D., Petway, L., Hines, G., and Roback, V., "Lidar systems for precision navigation and safe landing on Planetary Bodies," Proc. International Society for Optics and Photonics (SPIE), August 2011.

${ }^{10}$ Keim, J., Mobasser, S., Kuang, D., Cheng, Y., Ivanov, T., Johnson, A., Goldberg, H., Khanoyen, G., and Natzic, D., "Field test implementation to evaluate a flash LIDAR as a primary sensor for safe lunar landing," IEEE Aerospace Conference (AEROCONF 2010), March 2010.

${ }^{11}$ Amzajerdian, F., Pierrottet, D., Petway, L., Hines, G., and Barnes, B., "Doppler lidar sensor for precision navigation in GPS-deprived environment," Proc. International Society for Optics and Photonics (SPIE), June 2013.

${ }^{12}$ Pierrottet, D., Amzajerdian, F., and Barnes, B., "A long distance Laser Altimeter for terrain relative navigation and spacecraft landing," SPIE Defense and Security Symposium, June 2014.

${ }^{13}$ Trawny, N., Carson, J. M., Huertas, A., Luna, M. E., Roback, V. E., Johnson, A. E., Martin, K. E., and Villalpando, C. Y., "Helicopter Flight Testing of a Real-Time Hazard Detection System for Safe Lunar Landing," Proc. AIAA SPACE 2013 Conference \& Exposition, San Diego, CA, 10-12 Sept. 2013.

${ }^{14}$ Pierrottet, D. F., Amzajerdian, F., Petway, L. B., Hines, G. D., and Barnes, B., "Field Demonstration of a Precision Navigation Lidar System for Space Vehicles," Proc. AIAA Guidance, Navigation, and Control Conference, Boston, MA, August 2013.

${ }^{15}$ Rutishauser, D. K., Epp, C. D., and Robertson, E. A., "Free-Flight Terrestrial Rocket Lander Demonstration for NASA's Autonomous Landing and Hazard Avoidance Technology (ALHAT) System," Proc. AIAA SPACE 2012 Conference E3 Exposition, September 2012.

${ }^{16}$ Pierrottet, D., Amzajerdian, F., Petway, L., Barnes, B., and Lockard, G., "Flight test performance of a high precision navigation Doppler lidar," Proc. SPIE 7323, Laser Radar Technology and Applications XIV, Vol. 732311, doi:10.1117/12.821902, May 62009.

${ }^{17}$ Pierrottet, D., Amzajerdian, F., Petway, L., Lockard, B. B. . G., and Hines, G., "Navigation Doppler lidar sensor for precision altitude and vector velocity measurements: flight test results," Proc. SPIE 8044, Sensors and Systems for Space Applications IV, Vol. 80440S, doi:10.1117/12.886826, May 232011.

${ }^{18}$ Skolnik, M., Radar Handbook 2nd, Edition, McGraw-Hill Book Company, New York, 1990.

${ }^{19}$ Villalpando, C., Werner, R., III, J. C., Khanoyan, G., Stern, R., and Trawny, N., "A Hybrid FPGA/Tilera Compute Element for Autonomous Hazard Detection and Navigation," Proc. IEEE Aerospace Conference (AEROCONF 2013), March 2013.

${ }^{20}$ Carson, J. M., Bailey, E. S., Trawny, N., Johnson, A. E., Roback, V. E., Amzajerdian, F., and Werner, R. A., "Operations Concept, Hardware Implementation and Ground-Test Verification of a Hazard Detection System for Autonomous and Safe Precision Lunar Landing," Proc. AAS/AIAA Astrodynamics Specialist Conference, Hilton Head Island, SC, Aug. 11-15 2013.

${ }^{21}$ Roback, V. E., Bulyshev, A. E., Amzajerdian, F., Brewster, P. F., Barnes, B. W., Kempton, K. S., and Reisse, R. A., "Helicopter Flight Test of a Compact, Real-Time 3-D Flash Lidar for Imaging Hazardous Terrain during Planetary Landing," Proc. AIAA SPACE 2013 Conference \& Exposition, San Diego, CA, 10-12 Sept. 2013.

${ }^{22}$ Roback, V. E., Bulyshev, A. E., Amzajerdian, F., and Reisse, R. A., "Helicopter Flight Test of 3D Imaging Flash Lidar Technology for Safe, Autonomous, and Precise Planetary Landing," Proc. SPIE 8731, Laser Radar Technology and Applications XVIII, Vol. 87310H, doi:10.1117/12.2015961, June 4 2013, pp. 1-20.

${ }^{23}$ Stettner, R., Bailey, H., and Richmond, R., "Eye Safe Laser Radar Focal Plane Array for Three-Dimensional Imaging," Proc. International Society for Optics and Photonics (SPIE), 2001.

${ }^{24}$ Brady, T., Paschall, S., Crain, T., DeMars, K., and Bishop, R., "GENIE Flight Test Results and System Overview," Proc. AAS Guidance and Control Conference, February 2011. 